\title{
Fingering convection in red giants revisited
}

\author{
F. C. Wachlin ${ }^{1}$, S. Vauclair ${ }^{2,3}$, and L. G. Althaus ${ }^{1}$ \\ 1 Instituto de Astrofísica de La Plata (UNLP - CONICET), Facultad de Ciencias Astronómicas y Geofísicas, \\ Universidad Nacional de La Plata, 1900 La Plata, Argentina \\ e-mail: fcw@fcaglp.unlp.edu.ar \\ 2 Université de Toulouse, UPS-OMP, IRAP, 31400 Toulouse, France \\ 3 CNRS, IRAP, 14 avenue Edouard Belin, 31400 Toulouse, France
}

Received 10 July 2014 / Accepted 15 September 2014

\begin{abstract}
Context. Fingering (thermohaline) convection has been invoked for several years as a possible extra-mixing which could occur in red giant stars; it is due to the modification of the chemical composition induced by nuclear reactions in the hydrogen burning zone. Recent studies show, however, that this mixing is not sufficient to account for the needed surface abundances.

Aims. A new prescription for fingering convection, based on 3D numerical simulations has recently been proposed. The resulting mixing coefficient is larger than those previously given in the literature. We compute models using this new coefficient and compare them to previous studies.

Methods. We used the LPCODE stellar evolution code with a generalized version of the mixing length theory to compute red giant models and we introduce fingering convection using the BGS prescription.

Results. The results show that, although the fingering zone now reaches the outer dynamical convective zone, the efficiency of the mixing is not enough to account for the observations. The fingering mixing coefficient should be increased by two orders of magnitude for the needed surface abundances to be reached.

Conclusions. We confirm that fingering convection cannot be the mixing process needed to account for surface abundances in red giant branch stars.
\end{abstract}

Key words. instabilities - stars: evolution - stars: abundances - stars: interiors

\section{Introduction}

The formation and evolution of the chemical elements and their isotopic ratios in the Galaxy is a very rich and complex subject. At the present time, precise observations are obtained not only in stars with spectroscopic methods, but also in our close neighborhood, as a result of the detailed analyses of the presolar grains found inside meteorites and comets (Andersen \& Lattanzio 2007; Ott \& Hoppe 2007; Lugaro \& Höfner 2007; Nittler \& Alexander 2007). The element abundances and isotopic ratios measured in these grains may give information on the sites where these elements were processed. It has been shown that they come from evolved stars, red giant branch (RGB) and mostly asymptotic giant branch (AGB) stars. The comparisons between the observed abundance ratios and the abundance variations computed in stellar models show evidence that extramixing must occur in these stars as well as in standard convective zones (Nollett et al. 2003).

Evidence for the need of an extra-mixing process in RGB stars was already given by Charbonnel (1995). She pointed out that the observations of the carbon isotopic ratio in low mass red giant stars suggest that a mixing mechanism occurs below the convective zone, after the first dredge-up, at the moment when the hydrogen burning shell (HBS) reaches the region which was mixed during the dredge-up. Such a mixing could also partially destroy ${ }^{3} \mathrm{He}$, which would account for the observations of this element in HII regions, less abundant than expected from its formation in main-sequence stars.
Further computations by Palacios et al. (2006) showed that consistent computations of rotation-induced mixing in the framework of the shellular approximation (Zahn 1992) could not lead to a strong enough mixing to account for the observations. Eggleton et al. (2006) proposed that the mean molecular weight decrease induced by nuclear reactions in the hydrogen burning shell could lead to hydrodynamical instabilities and help account for the observations. They assumed, however, that the effect was dominated by Rayleigh-Taylor mixing, which is not the case. Charbonnel \& Zahn (2007) pointed out that thermohaline convection was the first process occurring in the presence of inverse $\mu$-gradients. They computed this effect using the prescription proposed by Ulrich (1972) for the mixing coefficient and found that the abundance observations could be nicely reproduced. Unfortunately, it was later proved by numerical simulations and analytical computations that the mixing coefficient they used was strongly overestimated (Denissenkov 2010; Traxler et al. 2011; Wachlin et al. 2011; Vauclair \& Théado 2012). Later on, an attempt was made to treat thermohaline and rotational-induced mixing together, by adding the corresponding mixing coefficients (Charbonnel \& Lagarde 2010; Lagarde et al. 2011). However, this very simple treatment did not take into account the influence of horizontal turbulence which would reduce the effect of the thermohaline process, as mentioned by Vauclair \& Théado (2012). This was later recognized by Maeder et al. (2013).

At the present time, we are in a situation where the presence of an extra mixing in red giants is clearly needed, but the reason 
for this mixing is still unknown (Denissenkov \& VandenBerg 2003; Busso et al. 2007; Denissenkov et al. 2009; Stancliffe 2010; Denissenkov \& Merryfield 2011). We also know that specific hydrodynamical processes in AGB stars are needed to explain the chemical evolution of the Galaxy (Denissenkov \& Tout 2003; Stancliffe et al. 2007; Thompson et al. 2008; Stancliffe et al. 2009).

In this paper, we focus on the subject of thermohaline mixing in RGB stars. Brown et al. (2013) performed new 3D simulations and gave a new 1D prescription of thermohaline convection (now preferably called "fingering convection" because "thermohaline" is more appropriate for the ocean than for stars). They gave evidence that the Traxler et al. (2011) treatment underestimated the mixing efficiency in the limits of very small Prandtl and Lewis numbers, which are characteristic of stellar conditions. It seemed important to test this new prescription for the red giant case. This was the motivation of the present paper. We present numerical computations of thermohaline convection using this new coefficient (Sect. 2). The results are given in Sect. 3 . We show that although the mixing is now clearly more efficient than when obtained with previous coefficients, it is not sufficient to lead to the needed abundance dilution of ${ }^{3} \mathrm{He}$. These results and their implications are discussed in Sect. 4.

\section{Numerical computations}

\subsection{Stellar models}

We computed the evolution of a $0.9 M_{\odot}$ model with initial metallicity of $[\mathrm{Fe} / \mathrm{H}]=-1.3$ from the zero-age main sequence (ZAMS) until the upper RGB, where the luminosity is $L \approx$ $10^{3} L_{\odot}$. We also computed stellar models with different metallicities, one with $[\mathrm{Fe} / \mathrm{H}]=-0.3$ (almost solar) and one with $[\mathrm{Fe} / \mathrm{H}]=-2.3$ to check the influence of the chemical composition on our final results. All calculations were done using the LPCODE stellar evolutionary code (Althaus et al. 2005, 2013). This is a well-tested and calibrated code that has been amply used in the study of different aspects of low-mass star evolution, including white dwarf stars. In particular, and for the relevance of the present paper, the code includes a generalized version of the mixing length theory developed by Grossman et al. (1993, hereafter GNA; see also Grossman \& Taam 1996). This double diffusive convection theory has already been successfully implemented in a similar context by Wachlin et al. (2011), and we refer the reader to this paper for details about the implementation of the GNA theory in our code. We set the GNA mixing length parameter to an equivalent value of $\alpha=1.66$ in the classical (MLT) theory. The use of the GNA convection theory allows us to infer the different unstable transport regimes, namely, dynamical convection, semi-convection, and fingering (thermohaline) convection, and to treat the corresponding mixing processes by using implemented diffusion coefficients.

Figure 1 shows the variations with depth of several parameters that are important for the computation of fingering convection in a $0.9 M_{\odot}$ model with $[\mathrm{Fe} / \mathrm{H}]=-1.3$. These parameters are the radiative viscosity $\nu_{\text {rad }}$, the molecular diffusivity $\kappa_{\mu}$, and the Prandtl and inverse Lewis numbers. The nuclear energy production is also presented in the same graph to highlight the hydrogen burning zone. This model corresponds to the moment when the fingering region reaches the bottom of the envelope dynamical convective zone (see, Sect. 3.1). If we compare this graph with the values given by Denissenkov (2010) in his Table 2, we see that they agree only in the lower part of the HBS. In this region the radiative viscosity $v_{\text {rad }}$ is of the same

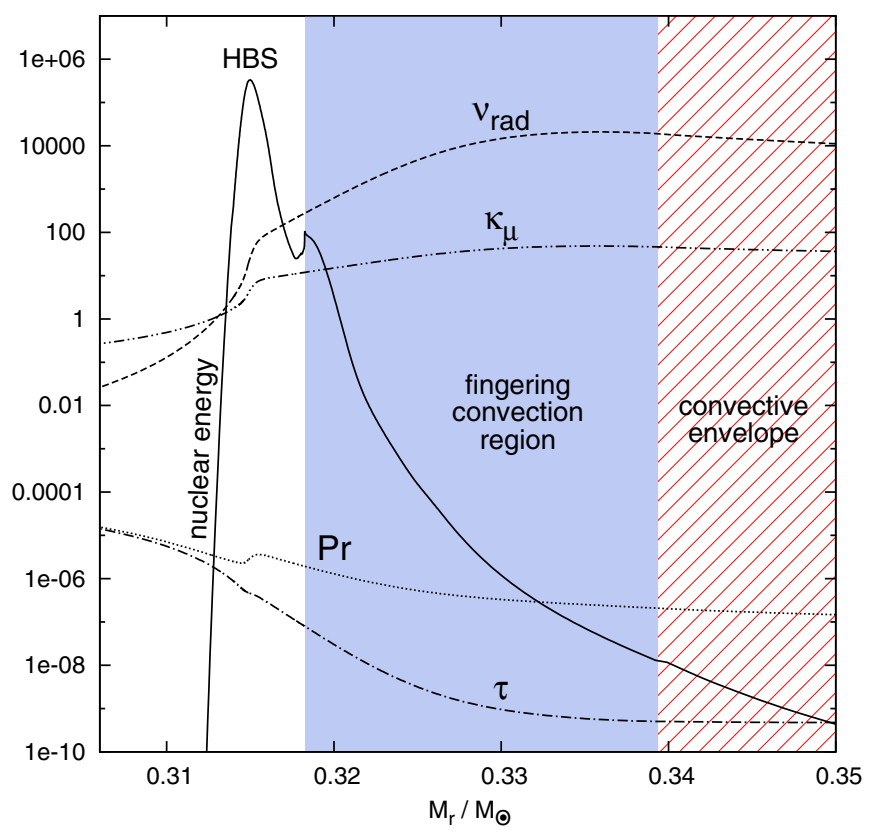

Fig. 1. Profiles of some relevant parameters for fingering convection as a function of the internal mass in a $0.9 M_{\odot}$ model with $[\mathrm{Fe} / \mathrm{H}]=-1.3$. The location of the HBS is also shown (nuclear energy is in $\operatorname{erg~g}^{-1} \mathrm{~s}^{-1}$ ). See text for details.

order as the molecular diffusivity $\kappa_{\mu}$, but their values are very different above and below these layers. At the place where the fingering convection actually develops, the radiative viscosity is more than two orders of magnitude larger than the molecular diffusivity, which strongly modifies the Prandtl number.

We also computed stellar models in which we included overshooting, following the prescription of Freytag et al. (1996). All convective boundaries are extended by assigning to that region an exponentially decaying diffusive coefficient of the form

$D=D_{0} \exp \left(-\frac{2 z}{f H_{p}}\right)$

where $D_{0}$ is the diffusive coefficient near the edge of the convective zone, $z$ denotes the distance of the considered layer to this edge, $H_{p}$ is the pressure scale height, and $f$ is a measure of the efficiency of the extra partial mixing. In the following, we explore three cases of overshooting: moderate $(f=0.015)$, intermediate $(f=0.075)$, and extreme $(f=0.15)$.

\subsection{Treatment of fingering convection}

In this paper we use the recent prescription given by Brown et al. (2013; hereafter BGS) for the computation of fingering convection, which represents a real improvement compared to the previous treatments (see also Zemskova et al. 2014).

Fingering (thermohaline) convection is a well-known process in the ocean. This instability occurs when hot salt water meets cool fresh water. It is at the origin of the global circulation in the Earth's oceans, and is called "thermohaline circulation". In stars, a similar instability occurs every time some upper layers have a higher mean molecular weight than deeper layers, in the presence of a stable temperature gradient. This may happen in the case of accretion of planetary matter (Vauclair 2004; Garaud 2011; Deal et al. 2013), or accretion of matter from a companion (Stancliffe \& Glebbeek 2008; Thompson et al. 2008), in the case of a local $\mu$-decrease due to nuclear reactions as in red giants 
F. C. Wachlin et al.: Fingering convection in red giants revisited

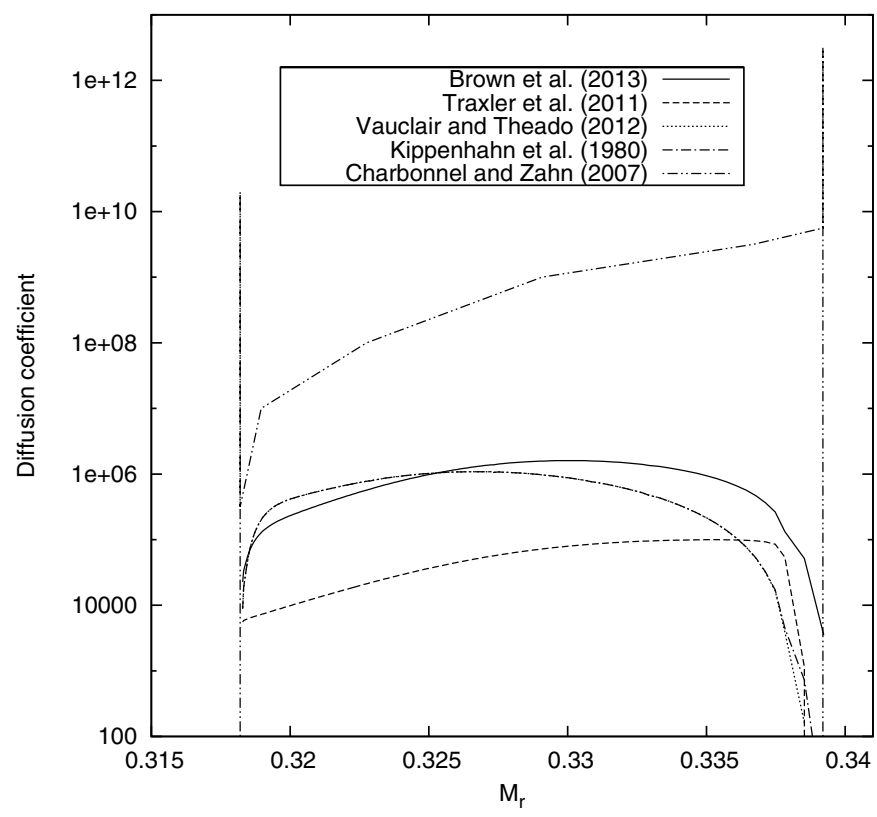

Fig. 2. Comparison of the diffusion coefficients for the fingering region corresponding to different prescriptions used in the past. The coefficients correspond to a stellar model at the time the fingering zone reaches the bottom of the dynamical convective zone when the BGS prescription is used (right panel of Fig. 3.)

(Charbonnel \& Zahn 2007), or in the presence of iron-rich layers induced by atomic diffusion (Théado et al. 2009; Zemskova et al. 2014).

The first treatments of fingering convection in stars were purely analytical (Ulrich 1972; Kippenhahn et al. 1980). They differed by orders of magnitude, according to the assumed shape of the fingers, which was unknown. Contrary to Vauclair (2004), Charbonnel \& Zahn (2007) used the Ulrich (1972) value, which is much larger than the Kippenhahn et al. (1980) one. More recently, 2D and 3D numerical simulations were performed, all converging on the result that the Ulrich (1972) value was strongly overestimated (Denissenkov 2010; Traxler et al. 2011). The new simulations by BGS including the evolution of the fingers with time, and the associated prescription, give coefficients slightly larger than the previous ones. The BGS coefficients are used in the present computations.

Figure 2 shows the coefficients obtained for various prescriptions in a $0.9 M_{\odot}$ model with $[\mathrm{Fe} / \mathrm{H}]=-1.3$. As will be seen below, the use of the BGS coefficient is enough for the fingering region to reach the bottom of the $\mathrm{CZ}$, but not enough to reduce ${ }^{3} \mathrm{He}$ significantly. Figure 2 corresponds to the time at which the fingering instability reaches the base of the convection zone when the BGS coefficient is used.

\section{Results}

\subsection{Behavior of the fingering convection zone}

We first describe the results obtained for a $0.9 M_{\odot}$ model with $[\mathrm{Fe} / \mathrm{H}]=-1.3$, without overshooting. We are mainly interested in the RGB phase around the luminosity bump, when the advance of the HBS over the homogeneous region left by the first dredge-up triggers fingering instability. This double-diffusive instability is closely related to the appearance of a compositional gradient inversion $\left(\nabla_{\mu}<0\right)$ soon after the luminosity bump.

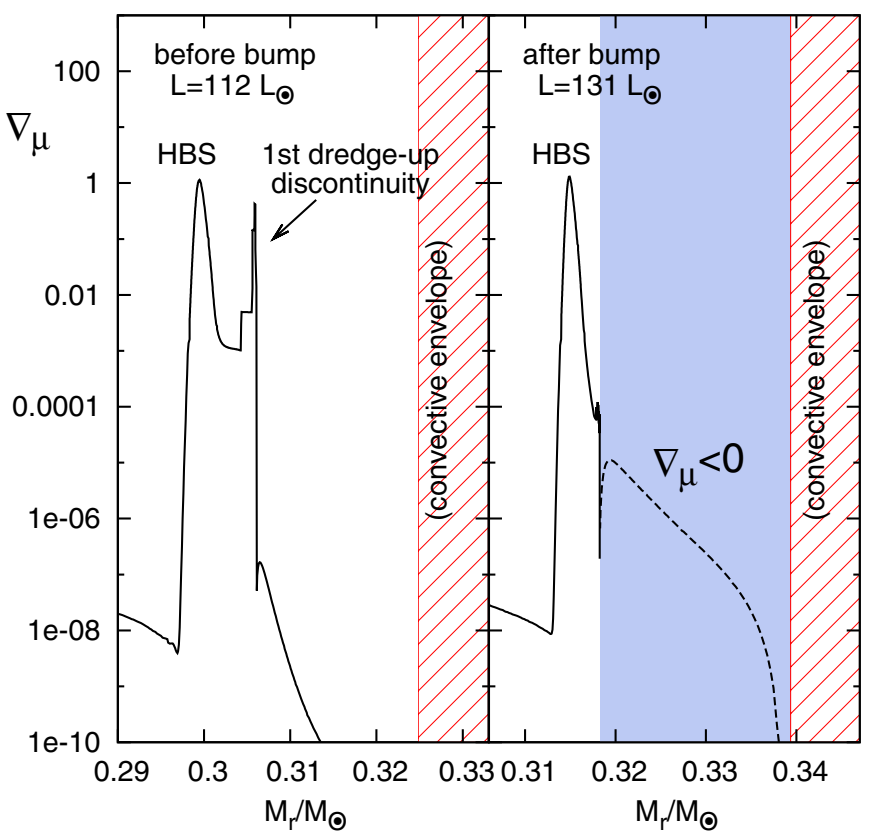

Fig. 3. Profile of the mean molecular weight gradient $\nabla_{\mu}$ as a function of mass. Solid line stands for $\nabla_{\mu}>0$ regions and the dashed line for all other cases. Left and right panels correspond to the situation before and after the bump, respectively, for the model of $0.9 M_{\odot},[\mathrm{Fe} / \mathrm{H}]=-1.3$. HBS: hydrogen burning shell; shaded zone: fingering unstable region; hatched area: innermost part of the convective envelope. The left and right panels are separated by $9.7 \mathrm{Myr}$ of evolution.

Figure 3 shows the compositional gradient profile near the location of the HBS for two evolutionary stages before (left panel) and after (right panel) the luminosity bump. In the left panel two peaks are apparent; the deeper peak (at $M_{r} \approx 0.3 M_{\odot}$ ) corresponds to the region where $\mathrm{H}$ is depleted by nuclear reactions in the HBS, whereas the second peak (at $M_{r} \approx 0.306 M_{\odot}$ ) corresponds to the chemical discontinuity at the point of maximum penetration of the first dredge-up. Since the HBS moves to the surface as $\mathrm{H}$ becomes exhausted, the situation corresponds to a moment shortly before the occurrence of the luminosity bump. No compositional gradient inversion is evident. In the right panel the situation is quite different. The panel illustrates the moment when the fingering instability region (shaded zone) first touches the convective envelope, 9.7 Myr after the situation shown in the left panel. It can be seen that the HBS has now reached the former homogeneous region. A compositional gradient inversion (dashed line) has developed between the HBS and the convective envelope. This $\nabla_{\mu}<0$ zone starts soon after the luminosity bump as a small region at the external wing of the HBS, but grows progressively until it reaches the receding convective envelope.

The most remarkable result here is that the unstable fingering zone eventually reaches the outer convection zone, which is a clear result of using the new Brown et al. (2013) prescription for the fingering convection. This never happened with the previous mixing coefficients, except the one used by Charbonnel \& Zahn (2007), which was strongly overestimated. This is the most important prediction of our simulations.

However, although the contact between both unstable regions might provide the extra-mixing mechanism that allows convective envelope material to reach the HBS and thus modify the surface abundances of ${ }^{3} \mathrm{He},{ }^{7} \mathrm{Li},{ }^{12} \mathrm{C},{ }^{13} \mathrm{C}$ and ${ }^{14} \mathrm{~N}$, no change in these abundances was found in this simulation. The reason is related to the efficiency of the thermohaline mixing, 


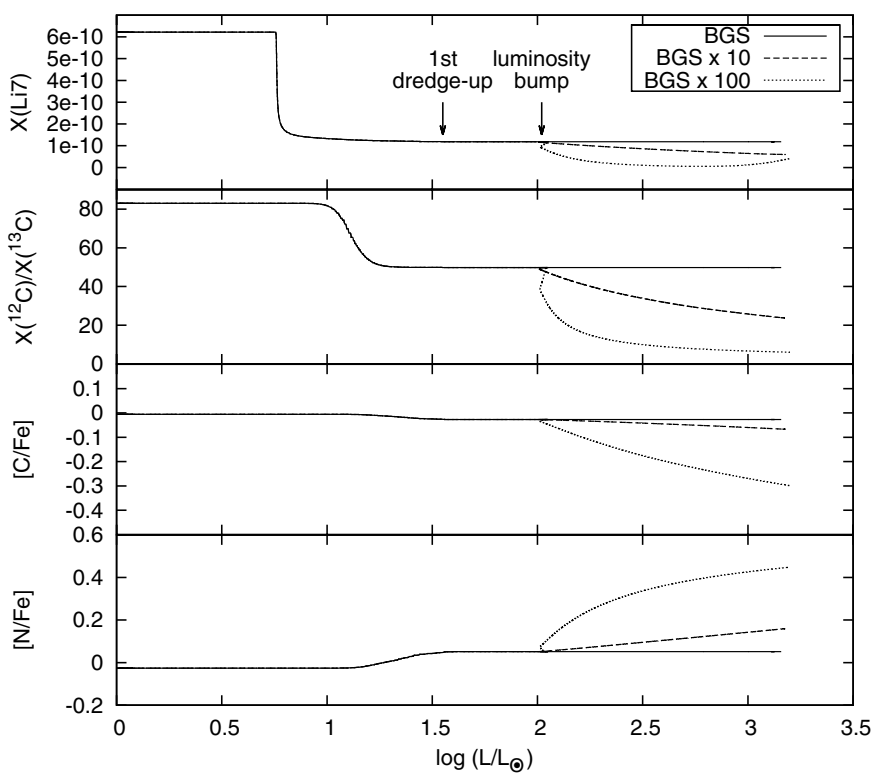

Fig. 4. Evolution of the surface lithium abundance, of the carbon isotopic ratio ${ }^{12} \mathrm{C} /{ }^{13} \mathrm{C}$, of $[\mathrm{C} / \mathrm{Fe}]$, and $[\mathrm{N} / \mathrm{Fe}]$ as a function of the luminosity logarithm for three simulations where the diffusion coefficient has been changed. The full line (labeled "BGS") was obtained implementing the Brown et al. (2013) prescription, the dashed line (labeled "BGS x 10") corresponds to the case where the diffusion coefficient is artificially increased by a factor of 10, and the dotted line (labeled "BGS x 100") refers to computation with a diffusion coefficient 100 times larger than that of Brown et al. (2013). The luminosity of the model at the moment of the first dredge-up and at the start of the luminosity bump are marked in the top panel.

which decreases according to the decrease in the $\mu$-gradient, so that when the mixed zone reaches the classical convective zone the fingering mixing efficiency becomes too weak to modify the abundances (see Figs. 4 and 5, full line labeled BGS).

\subsection{Influence of overshooting and different metallicities}

We have checked the possible influence of overshooting on the preceding results. Extending the envelope convective zone could actually lead to an overall more efficient mixing and help change the surface abundances as needed. We performed three new experiments, introducing a moderate $(f=0.015)$, an intermediate $(f=0.075)$, and a strong $(f=0.15)$ overshooting below the dynamical convective zone. No changes in the surface abundances were obtained in any of these three cases. The mixing efficiency of the fingering convection is much too low for the elements to be mixed between the convective envelope and the HBS, even in the presence of overshooting.

We also computed models with two different metallicities, $[\mathrm{Fe} / \mathrm{H}]=-0.3$ and $[\mathrm{Fe} / \mathrm{H}]=-2.3$. In both experiments the behavior of the fingering zone is very similar to that in the $[\mathrm{Fe} / \mathrm{H}]=-1.3$ case, although some small differences do appear. In the less metallic model the fingering region grows with a timescale of about $10 \mathrm{Myr}$ but never reaches the bottom of the convective zone, whereas for the more metallic model the fingering region comes in contact with the convective zone but, in this case, the surface abundances show a negligible change (for example, ${ }^{12} \mathrm{C} /{ }^{13} \mathrm{C}$ changes from 45.90 to 45.89 ).

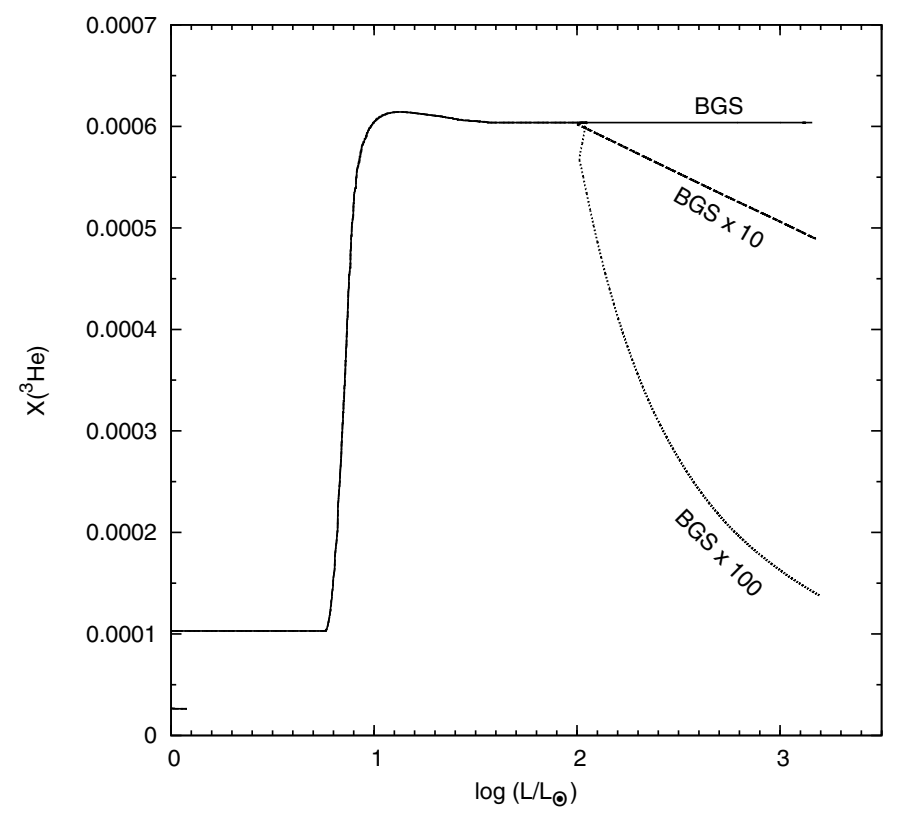

Fig. 5. Evolution of the surface abundance of the mass fraction of ${ }^{3} \mathrm{He}$. The labels of the curves are as in Fig. 4.

\subsection{Influence of artificially increasing the mixing coefficient}

As a toy model, we decided to explore the consequences of increasing the values proposed by Brown et al. (2013) in order to check the impact of such a modification on the observed surface abundances. We artificially increased the Brown et al. (2013) diffusion coefficients by factors of 10 and 100; no overshooting was introduced in these computations. Figure 4 shows the results, including the outcome of the simulation obtained with the original BGS diffusion coefficients.

An increase in the mixing efficiency of the fingering region by a factor of 10 is enough to modify the surface abundances of some elements, particularly the carbon isotopic ratio $\left({ }^{12} \mathrm{C} /{ }^{13} \mathrm{C}\right)$. Other quantities like the mass fraction of ${ }^{7} \mathrm{Li}$, or $[\mathrm{C} / \mathrm{Fe}]$ and $[\mathrm{N} / \mathrm{Fe}]$ present a small change at the luminosity bump $\left(\log L_{\text {bump }} / L_{\odot} \approx 2.05\right)$, and in the final abundances.

For a diffusion coefficient multiplied by 100 , the surface abundances change more rapidly than when multiplied by 10 . The final values are clearly different from the ones before the luminosity bump. The modification of the carbon isotopic ratio is particularly abrupt compared with the evolution of the other indicators.

Of particular interest is the evolution of the surface ${ }^{3} \mathrm{He}$ abundance for the different numerical experiments mentioned before. While no changes appear when we use the BGS prescription, the surface abundances are modified when we introduce larger mixing rates. Figure 5 shows the evolution of the ${ }^{3} \mathrm{He}$ surface abundance for the same simulations as presented in Fig. 4. In the classical picture, the surface ${ }^{3} \mathrm{He}$ composition changes as a consequence of the first dredge-up. After this episode, in the absence of any other mixing process, it should remain constant for the rest of the RGB evolution. Figure 5 shows that this is even the case for the implementation of the BGS prescription. However, as the mixing coefficient is increased, the surface ${ }^{3} \mathrm{He}$ mass fraction decreases as a consequence of its consumption in the HBS. For a diffusion coefficient multiplied by 10 , we see that $X\left({ }^{3} \mathrm{He}\right)$ decreases by a small amount, from $0.604 \times 10^{-3}$ to $0.490 \times 10^{-3}$, whereas for an increase by a factor 100 , the ${ }^{3} \mathrm{He}$ mass fraction rapidly decays to a final value of $0.137 \times 10^{-3}$, close to its initial 
abundance which is needed to reconcile the ${ }^{3} \mathrm{He}$ abundance with that observed in HII regions.

\section{Discussion and conclusion}

Since the Charbonnel \& Zahn (2007) paper in which fingering (thermohaline) convection was invoked as a possible explanation of the surface abundances in red giant stars, the description of this instability has much improved owing to 3D numerical simulations. It has been shown by several authors (Denissenkov 2010; Traxler et al. 2011; Vauclair \& Théado 2012) that the Ulrich prescription used at the beginning was strongly overestimated. All recent studies (see Wachlin et al. 2011) converge on the result that this extra mixing cannot account for the observations.

The present paper was motivated by the most recent 3D simulations of fingering convection and the derived 1D prescription of BGS. These new simulations and the new prescription give a better treatment than the previous ones for stellar conditions. The resulting mixing coefficient is larger and it was interesting to test its influence on the general results.

We have computed red giant models with several metallicities, with or without overshooting below the envelope dynamical convective zone. The important new result compared to previous studies is that, with this new prescription, the fingering zone induced by nuclear compositional changes may reach the bottom of the dynamical convective zone, which was not the case before. However, the efficiency of the added mixing induced by fingering convection is still two orders of magnitude too low to account for the observations.

We confirm in this paper that another kind of extra mixing is needed to account for the chemical composition of red giant and reconcile the production of ${ }^{3} \mathrm{He}$ with the Galactic observations.

Acknowledgements. This research was supported, in part by PIP 112200801-00940 from CONICET and by AGENCIA through the Programa de Modernización Tecnológica BID 1728/OC-AR. FCW and LGA would like to thank Marcelo M. Miller Bertolami for useful discussions about RGB stars and for major improvements made to LPCODE.

\section{References}

Althaus, L. G., Serenelli, A. M., Panei, J. A., et al. 2005, A\&A, 435, 631

Althaus, L. G., Miller Bertolami, M. M., \& Córsico, A. H. 2013, A\&A, 557, A19

Andersen, A. C., \& Lattanzio, J. C. 2007, Highlights of Astronomy, 14, 339

Brown, J. M., Garaud, P., \& Stellmach, S. 2013, ApJ, 768, 34

Busso, M., Wasserburg, G. J., Nollett, K. M., \& Calandra, A. 2007, ApJ, 671, 802

Charbonnel, C. 1995, ApJ, 453, L41

Charbonnel, C., \& Lagarde, N. 2010, A\&A, 522, A10

Charbonnel, C., \& Zahn, J.-P. 2007, A\&A, 476, L29

Deal, M., Deheuvels, S., Vauclair, G., Vauclair, S., \& Wachlin, F. C. 2013, A\&A, 557, L12

Denissenkov, P. A. 2010, ApJ, 723, 563

Denissenkov, P. A., \& Merryfield, W. J. 2011, ApJ, 727, L8

Denissenkov, P. A., \& Tout, C. A. 2003, MNRAS, 340, 722

Denissenkov, P. A., \& VandenBerg, D. A. 2003, ApJ, 593, 509

Denissenkov, P. A., Pinsonneault, M., \& MacGregor, K. B. 2009, ApJ, 696, 1823

Eggleton, P. P., Dearborn, D. S. P., \& Lattanzio, J. C. 2006, Science, 314, 1580

Freytag, B., Ludwig, H.-G., \& Steffen, M. 1996, A\&A, 313, 497

Garaud, P. 2011, ApJ, 728, L30

Grossman, S. A., \& Taam, R. E. 1996, MNRAS, 283, 1165

Grossman, S. A., Narayan, R., \& Arnett, D. 1993, ApJ, 407, 284

Kippenhahn, R., Ruschenplatt, G., \& Thomas, H.-C. 1980, A\&A, 91, 175

Lagarde, N., Charbonnel, C., Decressin, T., \& Hagelberg, J. 2011, A\&A, 536, A28

Lugaro, M. A., \& Höfner, S. 2007, Highlights of Astronomy, 14, 345

Maeder, A., Meynet, G., Lagarde, N., \& Charbonnel, C. 2013, A\&A, 553, A1

Nittler, L. R., \& Alexander, C. M. O. 2007, Highlights of Astronomy, 14, 357

Nollett, K. M., Busso, M., \& Wasserburg, G. J. 2003, ApJ, 582, 1036

Ott, U., \& Hoppe, P. 2007, Highlights of Astronomy, 14, 341

Palacios, A., Charbonnel, C., Talon, S., \& Siess, L. 2006, A\&A, 453, 261

Stancliffe, R. J. 2010, MNRAS, 403, 505

Stancliffe, R. J., \& Glebbeek, E. 2008, MNRAS, 389, 1828

Stancliffe, R. J., Glebbeek, E., Izzard, R. G., \& Pols, O. R. 2007, A\&A, 464, L57

Stancliffe, R. J., Church, R. P., Angelou, G. C., \& Lattanzio, J. C. 2009, MNRAS, 396, 2313

Théado, S., Vauclair, S., Alecian, G., \& Le Blanc, F. 2009, ApJ, 704, 1262

Thompson, I. B., Ivans, I. I., Bisterzo, S., et al. 2008, ApJ, 677, 556

Traxler, A., Garaud, P., \& Stellmach, S. 2011, ApJ, 728, L29

Ulrich, R. K. 1972, ApJ, 172, 165

Vauclair, S. 2004, ApJ, 605, 874

Vauclair, S., \& Théado, S. 2012, ApJ, 753, 49

Wachlin, F. C., Miller Bertolami, M. M., \& Althaus, L. G. 2011, A\&A, 533, A139

Zahn, J.-P. 1992, A\&A, 265, 115

Zemskova, V., Garaud, P., Deal, M., \& Vauclair, S. 2014, ApJ, submitted [arXiv: 1407.1437] 